\title{
Asymptotic quasi-completeness and ZFC
}

\author{
Mirna Džamonja* and Marco Panza ${ }^{\dagger}$
}

April 4, 2018

\begin{abstract}
The axioms ZFC of first order set theory are one of the best and most widely accepted, if not perfect, foundations used in mathematics. Just as the axioms of first order Peano Arithmetic, ZFC axioms form a recursively enumerable list of axioms, and are, then, subject to Gödel's Incompleteness Theorems. Hence, if they are assumed to be consistent, they are necessarily incomplete. This can be witnessed by various concrete statements, including the celebrated Continuum Hypothesis CH. The independence results about the infinite cardinals are so abundant that it often appears that ZFC can basically prove very little about such cardinals.

However, we put forward a thesis that ZFC is actually very powerful at some infinite cardinals, but not at all of them. We have to move away from the first few and to look at limits of uncountable cardinals, such as $\aleph_{\omega}$. Specifically, we work with singular cardinals (which are necessarily limits) and we illustrate that at such cardinals there is a very serious limit to independence and that many statements which are known to be independent on regular cardinals become provable or refutable by ZFC at singulars. In a certain sense, which we explain, the behavior of the set-theoretic universe is asymptotically determined at singular cardinals by the behavior that the universe assumes at the smaller regular cardinals. Foundationally, ZFC provides an asymptotically univocal image of the universe of sets around the singular cardinals. We also give a philosophical view accounting for the relevance of these claims in a platonistic perspective which is different from traditional mathematical platonism.
\end{abstract}

\section{Introduction}

Singular cardinals have a fascinating history related to an infamous event in which one mathematician tried to discredit another and ended up being himself proved wrong. As Menachem Kojman states in his historical article on singular cardinals [28], 'Singular cardinals appeared on the mathematical world stage two years before they were defined'. In a public lecture at the Third International Congress of Mathematics in 1904, Julius König claimed to have proved that the continuum could not be well-ordered, therefore showing that Cantor's Continuum Hypothesis does not make sense, since this would entail that $2^{\aleph_{0}}$, the (putative) cardinal of the continuum, is not well defined. This was not very pleasant for Cantor, who was not alerted in advance and who was in the audience. However, shortly after, Felix Hausdorff found a mistake in König's

* School of Mathematics, University of East Anglia, Norwich, UK.

${ }^{\dagger}$ CNRS, IHPST (CNRS and Université Paris 1, Panthéon-Sorbonne) and Chapman University, Orange, CA. 
reasoning, which was to have used an incorrect lemma of Bernstein, proved by induction on all $\aleph_{\mathrm{s}}$ by an argument that did not work at an uncountable $\aleph$ that happens not to have an immediate predecessor. Such cardinals were then named 'singulars' by König in 1905.

This is not the way we define singular cardinals today. The difference between the modern definition and König's has important consequences that deserve an explanation.

Today, in ZFC, we regard cardinals as initial ordinals and the inherited order lets us talk about the cofinality of a cardinal $\kappa$, namely $\operatorname{cf}(\kappa)$. In ZF, following von Neumann, an ordinal is defined to be a transitive set well-ordered by the membership relation. It can then be seen that each ordinal is exactly the set of ordinals strictly smaller than it. A cardinal is an ordinal that is not bijective with any ordinal smaller than it. For example, $\omega$ is a cardinal since it is the smallest infinite ordinal. Notice that every ordinal $\alpha$ is bijective with exactly one cardinal, which we denote by ' $|\alpha|$ ' and call 'the cardinality of $\alpha$ '.

As such, the definition of the cardinality of an ordinal does not require the Axiom of Choice, but this axiom is required to ensure that every set has a cardinality. More precisely, in ZF one can prove that every well-ordered set is bijective with exactly one cardinal, but not that any set can be well-ordered. This is what the Axiom of Choice states (in one of its equivalent forms).

A set $B$ is cofinal in an ordered set $(A, \leq)$ if and only if $B$ is a subset of $A$ and for any $x$ in $A$ there is a $y$ in $B$ such that $x \leq y$. The cofinality $\operatorname{cf}(A)$ of an ordered set $A$ is the smallest cardinality of a set cofinal in $A$. This definition applies in particular when $A$ is a cardinal $\kappa$ considered as an ordered set with the relation $\leq$ between ordinals, giving us the definition of $\mathrm{cf}(\kappa)$. Any cardinal is obviously cofinal in itself. Hence, from the definition above it immediately follows that, for any cardinal $\kappa, \operatorname{cf}(\kappa) \leq \kappa$. Given this, we say that a cardinal $\kappa$ is regular if and only if $\operatorname{cf}(\kappa)=\kappa$. We say, then, that $\kappa$ is singular just if it is not regular. For example, $\aleph_{\omega}$ and $\aleph_{\omega+\omega}$ and $\aleph_{\omega_{1}}$ are each singular.

Already in ZF, it can be easily shown that the class of ordinals is well-ordered by the membership relation. It follows that every cardinal has an immediate successor. If the former is $\lambda$, we denote the latter by ' $\lambda^{+}$'. A cardinal which has an immediate predecessor is said to be a successor cardinal. If a cardinal is not a successor, then we say that it is a limit cardinal.

The example of $\aleph_{0}$ is enough to show that a limit cardinal can be regular. While analyzing König's attempted proof, Hausdorff proved, appealing to the Axiom of Choice, that all successor cardinals are regular. This makes, for example, $\aleph_{n}$ regular for any natural $n$. But this cannot be proved without the Axiom of Choice. In the absence of Choice it may even happen that all uncountable cardinals are singular. Indeed, Motik Gitik ([18]) has given a model of ZF where all uncountable cardinals are not only singular, but they all also have cofinality $\aleph_{0}$ (the model is described in some detail at the end of §3). In addition, even if the Axiom of Choice is admitted, we cannot prove just within ZFC, that there are cardinals other than $\aleph_{0}$ and 0 which are both limit and regular, that is, weakly inaccessible, as any such putative cardinal is usually called (this follows from Gödel's incompleteness). On the other hand, it is easy to see that there are unboundedly many singular cardinals. For example, $\aleph_{\alpha+\omega}$ is singular for any ordinal $\alpha$.

All this came about since the existence of singular cardinals destroyed an infamous argument put forward in 1904. But there is little justification in the qualification 'singular' that was obtained because of this incident, since there are unboundedly many such cardinals. This qualification is even quite unfortunate because, in fact, it is at these cardinals that set theory behaves better: it turns out that at them the incompleteness phenomena of ZFC are much less present (although not totally absent) than at regular cardinals. We shall illustrate this fact by various recent math- 
ematical findings. These results show that many statements which are known to be independent at regular cardinals become provable or refutable by ZFC at singulars, and so indicate that the behavior of the set-theoretic universe is asymptotically determined at singular cardinals by its features at the smaller regular cardinals. We could say, then, that even though ZFC is provably incomplete, asymptotically, at singular cardinals, it becomes quasi-complete since the possible features of universes of ZFC are limited in number, relative to the size of the singular in question. These facts invite a philosophical reflection.

The paper is organized as follows: Mathematical results that illustrate the mentioned facts are expounded in sections $\S 2$ and $\S 3$. The former contains results that by now are classic in set theory and it is written in a self-contained style. The latter contains results of contemporary research and is meant to reinforce the illustration offered by the former. This section is not written in a self-contained style, and it would be out of the scope of this paper to write it in this way. Section $\S 2$ also contains a historical perspective. Finally, some philosophical remarks are made in $\S 4$.

\section{Modern history of the singular cardinals}

One of the most famous (or infamous, depending on the point of view) problems in set theory is that of proving or refuting the Continuum Hypothesis $(\mathrm{CH})$ and its generalisation to all infinite cardinals $(\mathrm{GCH})$.

Cantor recursively defined two hierarchies of infinite cardinals, the $\aleph_{s}$ and the $\beth$ s, the first based on the successor operation and the second on the power set operation: $\aleph_{0}=\beth_{0}=\omega$, $\aleph_{\alpha+1}=\aleph_{\alpha}^{+}, \beth_{\alpha+1}=2^{\beth_{\alpha}}$, and for $\delta$ a non-zero limit ordinal $\aleph_{\delta}=\sup _{\beta<\delta} \aleph_{\beta}, \beth_{\delta}=\sup _{\beta<\delta} \beth_{\beta}$ (here we are using the notation ' $\sup (\mathcal{A})$ ' for a set $\mathcal{A}$ of cardinals to denote the first cardinal greater or equal to all cardinals in $\mathcal{A}$ ). A simple way to state GCH is to claim that these two hierarchies are the same: $\aleph_{\alpha}=\beth_{\alpha}$, for any $\alpha$. Another way, merely involving the first hierarchy, is to claim that for every $\alpha$ we have $2^{\aleph_{\alpha}}=\aleph_{\alpha}^{+}$. CH is the specific instance $\aleph_{1}=\beth_{1}$ or $2^{\aleph_{0}}=\aleph_{1}$. Insofar as $\beth_{1}=|\mathbb{R}|$, CH can be reformulated as the claim that any infinite subset of the set of the real numbers admits a bijection either with the set of natural numbers or with the set of real numbers.

It is well known that, frustratingly, Cantor spent at least thirty years trying to prove $\mathrm{CH}$. Hilbert choose the problem of proving or disproving GCH as the first item on his list of problems presented to the International Congress of Mathematics in 1900. In 1963 ([8]), Paul Cohen proved that the negation of $\mathrm{CH}$ is relatively consistent with ZFC. This result, jointly with that proved by Kurt Gödel in 1940 ([20]) - that GCH is also relatively consistent with ZFC - entails that neither $\mathrm{CH}$ nor GCH are provable or refutable from the axioms of ZFC.

Cohen's result came many years after Gödel's incompleteness theorems ([19]), which imply that there is a sentence in the language of set theory whose truth is not decidable by ZFC. But the enormous surprise was that there are undecidable sentences which are not specifically constructed as a Gödel's sentence; in particular, there is one as simply stated and well known as $\mathrm{CH}$.

There are many mathematical and philosophical issues connected to this outcome. The one which interests us here concerns the consequences it has for ZFC's models: it entails that if ZFC is consistent at all, then it admits a huge variety of different models, where CH and CGH are either true or false and, more generally, the power set class-function (namely $F: \mathfrak{R e g} \rightarrow \mathfrak{R e g} ; F(\kappa)=2^{\kappa}$, where $\mathfrak{R e g}$ is the class of regular cardinals) behaves in almost arbitrary ways (see below on the results of William Easton). This means that ZFC's axioms leave the von Neumann universe of 
sets $V$-which is recursively defined by appealing to the power set operation $\left(V=\bigcup_{\alpha} V_{\alpha}\right.$, with $\alpha$ an ordinal and $V_{\alpha}=\bigcup_{\beta<\alpha} \mathcal{P}\left(V_{\beta}\right)$ )-hugely indeterminate: they are compatible, for example, both with the identification of $V$ with Gödel's constructible universe $L$ (which is what the axiom of constructibility ' $V=L$ ' asserts, by, then, deciding GCH in the positive), and with the admission that in $V$ the values of $2^{\kappa}$ are as large as desired, which makes $V$ hugely greater than $L$. The question is whether this indetermination of the size of $V_{\alpha}$ versus the size of $L_{\alpha}$ can be somehow limited for some sort of cardinals, i.e. for some values of $\alpha$. The results we mention below show that this is so for singular cardinals, and even, as we said above, that $V$ is asymptotically determined at singular cardinals by its features at the smaller regular cardinals.

To explain this better, we begin with a result by Easton ([16]), who, shortly after Cohen's result and building on earlier results of Robert Solovay ([45]), proved that for regular cardinals the indetermination of the values of the power set function is even stronger than the Cohen's result suggests: for any non-decreasing class-function $F: \mathfrak{R e g} \rightarrow \mathfrak{R e g}$ defined in an arbitrary model of ZFC so that $\operatorname{cf}(F(\kappa))>\kappa$ for all $\kappa$, there is an extension to another model that preserves both cardinals and cofinalities and in which $2^{\kappa}=F(\kappa)$, for any regular cardinal $\kappa$. This implies that in ZFC no statement about the power set (class)-function ${ }^{1}$ on the regular cardinals other than ' $\kappa \leq \lambda \Longrightarrow 2^{\kappa} \leq 2^{\lambda}$ ' and 'cf $(\kappa)<\operatorname{cf}\left(2^{\kappa}\right)$ ' can be proved.

It is important to notice that singular cardinals are excluded from Easton's result. Just after the result was obtained, it was felt that this restriction was due to a technical problem which could be overcome in the future. But what became clear later is that this restriction is due to deep differences between regular and singular cardinals. Indeed, many results attesting to this soon followed. In particular, what these results eventually showed is that the power set classfunction behaves much better at singular cardinals than it does at regular ones. While the above quoted results by Gödel, Cohen and Easton imply that the value of the power set function can be decided in ZFC for neither regular nor singular cardinals, as not even $2^{\aleph_{0}}$ has an upper bound there, it turns out that one can do the next-best thing and show in ZFC that the value of $2^{\kappa}$ for any singular $\kappa$ is conditioned on the values of $2^{\lambda}$ for the regular $\lambda$ less than $\kappa$. This entails that the size of $V_{\kappa+1}$ is, in turn, conditioned by that of that of $V_{\lambda}$ for $\lambda \leq \kappa$.

Already by 1965 and 1973 respectively, Lev Bukovský ([5]) and Stephen H. Hechler ([21]) had proved, for example, that in ZFC if $\kappa$ is singular and $2^{\lambda}$ is eventually constant for $\lambda<\kappa$, then $2^{\kappa}$ is equal to this constant. Therefore the value of $2^{\kappa}$ is entirely determined by the values of the power set function below $\kappa$. An infinite cardinal $\lambda$ is said to be strong limit if for any $\theta<\lambda$ we have $2^{\theta}<\lambda$ (in particular, it follows that such a cardinal is limit). Note that strong limit cardinals, and in particular, strong limit singular cardinals, exist in any universe of set theory: an example is given by $\beth_{\omega}$. Solovay ([46]) proved that for any $\kappa$ which is larger or equal to a strongly compact cardinal (a large cardinal $\lambda$ characterised by having a certain algebraic property that is not essential to explain here, namely that any $\lambda$-complete filter can be extended to a $\lambda$-complete ultrafilter), we have $2^{\kappa}=\kappa^{+}$. In other words, GCH holds above a strongly compact cardinal. This result, of course, is only interesting if there exists a strongly compact cardinal. In fact this result was obtained as part of an investigation started earlier by Dana Scott [36], who investigated the question of what kind of cardinal can be the first cardinal failing GCH, that is, what properties

\footnotetext{
${ }^{1}$ According to the common abuse of notation, we call $F$ 'power set function', even though it is in fact a classfunction.
} 
must have a cardinal $\kappa$ such that $2^{\kappa}>\kappa^{+}$, but such that $2^{\theta}=\theta^{+}$, for all infinite cardinals $\theta<\kappa$. What Solovay's result shows is that such a cardinal cannot be strongly compact.

This result led Solovay to advance a new hypothesis, according to which, for singular cardinals, his own result does not depend on the existence of a strongly compact cardinal. In other words, the hypothesis is that in ZFC, every singular strong limit cardinal $\kappa$ satisfies $2^{\kappa}=\kappa^{+}$. The heart of it is the following implication called the 'Singular Cardinal Hypothesis':

$$
2^{\operatorname{cf}(\kappa)}<\kappa \Longrightarrow \kappa^{\operatorname{cf}(\kappa)}=\kappa^{+},
$$

for any cardinal $\kappa$. Indeed, for definition, the antecedent implies that $\kappa$ is a singular cardinal, so that $\mathrm{SCH}$ states that $\kappa^{\mathrm{cf}(\kappa)}=\kappa^{+}$, for any singular cardinal $\kappa$ for which this is not already ruled out by $2^{\mathrm{cf}(\kappa)}$ being too big. On the other hand, if $\kappa$ is a strong limit cardinal, then it follows from the elementary results mentioned in the previous section that $\kappa^{\mathrm{cf}(\kappa)}=2^{\kappa}$ (see [27], pg. 55), so that the consequent reduces to ' $2^{\kappa}=\kappa^{+}$'. Hence, $\mathrm{SCH}$ implies that the power set operation is entirely determined on the singular strong limit cardinals, since GCH holds for any such cardinal.

In a famous paper appearing in 1975 ([44]), Jack Silver proved that if $\kappa$ is a singular cardinal of uncountable cofinality, then $\kappa$ cannot be the first cardinal to fail GCH. A celebrated and unexpected counterpart of this result was proved by Menachem Magidor shortly afterwards ([31]). It asserts that in the presence of some rather large cardinals, it is consistent with ZFC to assume that $\aleph_{\omega}$ is the first cardinal that fails GCH. This, of course, implies that the condition that $\kappa$ has uncountable cofinality is a necessary condition for Silver's result to hold. But it also implies that $\mathrm{SCH}$ fails and that the power set function at the strong limit singular cardinals does not always behave in the easiest possible way.

Another celebrated theorem proved shortly after the work of Silver is Jensen's Covering Lemma ([11]), from which it follows that if there are no sufficiently large cardinals in the universe, then $\mathrm{SCH}$ holds. To be precise, this lemma implies that $\mathrm{SCH}$ holds if $0^{\sharp}$ does not exist. (It is probably not necessary here to define $0^{\sharp}$, but let us say that it is a large cardinal whose existence would make $V$ be larger than $L$, whereas its nonexistence would make $V$ be closely approximated by $L$.)

Further history of the problem up to the late 1980s is quite complex and involves notions that are out of the scope of ZFC and, a fortiori out of the scope of our paper. Details can be found, for example, in the historical introduction to [43]. Insofar as our interest here is to focus on the results that can be proved in ZFC, we confine ourselves to mention a surprising result proved by Fred Galvin and András Hajnal in 1975 ([17]). By moving the emphasis from GCH to the power set function as such, they were the first to identify a bound in ZFC for a value of this function, namely for the value it would take on a strong limit singular cardinal with uncountable cofinality. Let $\kappa$ be such a cardinal, then what Galvin and Hajnal proved is that $2^{\kappa}<\aleph_{\gamma}$, where $\gamma=\left(2^{|\alpha|}\right)^{+}$for that $\alpha$ for which $\kappa=\aleph_{\alpha}$. As the comparison with the two results of Silver and Magidor mentioned above makes clear, singular cardinals with countable and uncountable cofinality behave quite differently. There were no reasons in principle, then, to think, that Galvin and Hajnal's result would extend to singular cardinals with countable cofinality and the state of the matters stood still for many years.

Fast forward, and we arrive at a crowning moment in our story, namely to the proof, by Saharon Shelah in the late 1980s, of the following unexpected theorem, put forward in [43]:

$$
\left[\forall n\left(n<\omega \Longrightarrow 2^{\aleph_{n}}<\aleph_{\omega}\right)\right] \Longrightarrow 2^{\aleph_{\omega}}<\aleph_{\omega_{4}} .
$$


Shelah's theorem is, in fact, more general than the instance we quoted, which nevertheless perfectly illustrates the point. If $\aleph_{\omega}$ is a strong limit, then the value of the power set function on it is bounded. In every model of ZFC, Shelah's theorem extends to the countable cofinality the result of Galvin and Hajnal, obtains a bound in terms of just the $\aleph$-function (unlike the Galvin-Hajnal theorem which uses the power set function), and shows that in spite of Magidor's result (which shows that SCH can fails at singular strong limits cardinals of countable cardinality), even at such cardinals a weak form of SCH holds, namely the value of the power set function is bounded.

Shelah's theorem is proved by discovering totally new operations on cardinals, called 'pcf' and 'pp', which are meaningful for singular cardinals and whose values are very difficult to change by forcing. In many instances it is not even known if they are changeable to any significant extent. It would be much too complex for us to describe these operations here but the point made is that even though ZFC axioms are quite indecisive about the power set operation in general, they are quite decisive about it at the singular cardinals and this is because they prove deep combinatorial facts about the operations pcf and pp. The field of research concerned with the operations pcf and pp is called the 'pcf theory'.

\section{Some contemporary results}

The foregoing results have been known to mathematicians for a while but they do not seem to have influenced the literature in philosophy very much. The purpose of this article is to suggest that they have some interest for our philosophical views about ZFC and, more generally, set theory. Before coming to it, however, let us make a short detour in the realm of some more recent results which further illustrate the point. These results, to which this section is devoted, deal with mathematical concepts which are rather advanced; it would distract from the point to present them in a self-contained manner. Those readers who are not at ease with these concepts can safely skip the present section, taking it on trust that contemporary research continues to prove that singular cardinals have quite peculiar features, and that the mathematical universe at such cardinals exhibits much less indetermination than at the regular cardinals. This is the view that we shall discuss in $\S 4$.

Let us begin by observing that the emphasis of the recent research on singular cardinals has moved from cardinal arithmetic to more combinatorial questions. We could say that what recent research on singular cardinals is concerned with is combinatorial SCH: rather than just looking at the value of $2^{\kappa}$ for a certain cardinal $\kappa$, one considers the "combinatorics" of $\kappa$, namely the interplay of various appropriate properties $\varphi(\kappa)$ of it. An example of such a property might be the existence of a certain object of size $\kappa$, such as a graph (see below on graphs) on $\kappa$ with certain properties, or the existence of a topological or a measure-theoretic object of size $\kappa$, in the more complex cases. One may think of $\kappa$ as a parameter here. Then the relevant instance of combinatorial SCH would say that the property $\varphi(\kappa)$ depends only on the fact that $\varphi(\theta)$ holds at all $\theta<\kappa$. The question can be asked more generally, what about the relevant property of $\kappa$ can be proved in ZFC, knowing that the property holds all $\theta<\kappa$.

Concerning the former aspect of such a question, that concerned with what can be proved in ZFC, a celebrated singular compactness theorem has been proved by Shelah in [40]. Shelah's book [43] presents, moreover, many applications of pcf theory to deal with this aspect of the question. The latter aspect of the question-namely the forcing counterparts of the former-appeared only 
later, due to the enormous difficulty of doing even the simplest forcing at a singular cardinal and the necessity (by the Covering Lemma) of using large cardinals, for performing this task. One of the early examples is [14].

To illustrate this sort of research, let us concentrate on one sample combinatorial problem, which has to do with one of the simplest but most useful notions in mathematics, that of a graph.

A graph is a convenient way to represent a binary relation. Namely, a graph $(V, E)$ consists of a set $V$ of vertices and a set $E \subseteq V \times V$ of edges. Both finite and infinite graphs are frequently studied in mathematics and they are also used in everyday life, for example to represent communication networks. Of particular interest in the theory of graphs is the situation when one graph $G$ is subsumed by another one $H$, in the sense that one can find a copy of $G$ inside of $H$. This is expressed by saying that there is an embedding from $G$ to $H$. Mathematically speaking, this is defined as follows.

Definition 1 Suppose that $G=\left(V_{G}, E_{G}\right)$ and $H=\left(V_{H}, E_{H}\right)$ are graphs and $f: G \rightarrow H$ is a function. We say that $f$ is a graph homomorphism, or a homomorphic embedding if $f$ preserves the edge relation (so a $E_{G} b$ implies $f(a) E_{H} f(b)$ for all $a, b \in V_{G}$ ) but it is not necessarily 1-1. If $f$ is furthermore 1-1, we say that $f$ is a weak embedding. If, in addition, $f$ preserves the non-edge relation (so a $E_{G} b$ holds iff $f(a) E_{H} f(b)$ holds), we say that $f$ is a strong embedding.

Graph homomorphisms are of large interest in the theory of graphs and theoretical computer science (see for example [23] for a recent state-of-the-art book on graph homomorphisms). The decision problem associated to the graph homomorphism, that is, deciding if there is a graph homomorphism from one finite graph into another, is NP-complete (see Chapter 5 of [23], which makes the notion also interesting in computer sciences).

Of particular interest in applications is the existence of a universal graph. If we are given a class $\mathcal{G}$ of graphs, we say that a certain graph $G^{*}$ is universal for $\mathcal{G}$ if every graph from $\mathcal{G}$ admits a homomorphic embedding into $G^{*}$. Of course, variants of this relation can be obtained by replacing homomorphic embedding with weak or strong embedding, as defined in Definition 1. The combinatorial question that we shall survey is that of the existence of universal graphs of a fixed size $\kappa$ in various contexts.

To begin with, ZFC proves that there is a unique up to isomorphism graph $G^{*}$ of size $\aleph_{0}$. This is known as a Rado graph (or, also, random or Erdös-Rényi graph), and it satisfies that for every finite graph $G$ and every vertex $v$ of $G$, every strong embedding of $G \backslash\{v\}$ into $G^{*}$ can be extended to a strong embedding of $G$ into $G^{*}$. As a consequence, $G^{*}$ strongly embeds all countable graphs. This graph was discovered independently in several contexts, starting from the work of Ackermann in [2], but its universality properties were proved by Rado in [33].

Under the assumption of GCH, from the existence of saturated and special models in firstorder model theory (see [7]), it follows that a universal graph exists at every infinite cardinal $\kappa$. In particular, the assumption that $\lambda<\kappa \Longrightarrow \kappa^{\lambda}=\kappa$ entails that there is a saturated, and consequently universal, graph of size $\kappa$.

When we move away from GCH, the existence of universal graphs becomes a rather difficult problem. Shelah mentioned in [41] a result of his (for the proof see [30] or [12]), namely that adding $\aleph_{2}$ Cohen reals to a model of $\mathrm{CH}$ destroys any hope of having a universal graph of size $\aleph_{1}$. This does not only mean that there is no universal graph in this model, but also that, by defining the universality number of a family $\mathcal{G}$ of graphs as the smallest size of a subfamily $\mathcal{F}$ of $\mathcal{G}$ such that every element of $\mathcal{G}$ embeds into a member of $\mathcal{F}$, we have that in the above model the 
universality number of the family of graphs of size $\aleph_{1}$ is the largest possible, namely $2^{\aleph_{1}}$. More generally, one can state the following theorem:

Theorem 2 [Shelah, see [30] or [12]] Suppose that $\lambda<\kappa \Longrightarrow \kappa^{\lambda}=\kappa$ and let $\mathbb{P}$ be the forcing to add $\lambda$ many Cohen subsets to $\kappa$ (with $\operatorname{cf}(\lambda) \geq \kappa^{++}$and $\lambda \geq 2^{\kappa^{+}}$). Then the universality number for graphs on $\kappa^{+}$in the extension by $\mathbb{P}$ is $\lambda$.

Using a standard argument about Easton forcing, we can see that it is equally easy to get negative universality results for graphs at a class of regular cardinals:

Theorem 3 Suppose that the ground model $V$ satisfies $G C H$ and $\mathcal{C}$ is a class of regular cardinals in $V$, while $F$ is a non-decreasing function on $\mathcal{C}$ satisfying that for each $\kappa \in \mathcal{C}$ we have $\operatorname{cf}(F(\kappa)) \geq$ $\kappa^{++}$. Let $\mathbb{P}$ be Easton's forcing to add $F(\kappa)$ Cohen subsets to $\kappa$ for each $\kappa \in \mathcal{C}$. Then for each $\kappa \in \mathcal{C}$ the universality number for graphs on $\kappa^{+}$in the extension by $\mathbb{P}$ is $F(\kappa)$.

The proofs of these results are quite easy. In [41], Shelah emphasizes this by claiming that "The consistency of the non-existence of a universal graph of power $\aleph_{1}$ is trivial, since, it is enough to add $\aleph_{2}$ generic Cohen reals". He focuses, indeed, on a much more complex proof, that of the consistency of the existence of a universal graph at $\aleph_{1}$ with the negation of $\mathrm{CH}$. He obtained such a proof in [42], while Mekler obtained a different proof of the same fact in [32]. Insofar as $\aleph_{0}$ is regular, $\aleph_{1}$ is the successor of a regular cardinal. Other successors of regular cardinals behave in a similar way, although neither Mekler's nor Shelah's proof seems to carry over from $\aleph_{1}$ to larger successors of regulars. A quite different proof, applicable to larger successors of regulars but proving a somewhat weaker statement, was obtained by Džamonja and Shelah in [15]: they proved that assuming that it is relatively consistent with ZFC that the universality number of graphs on $\kappa^{+}$for an arbitrary regular $\kappa$ is equal to $\kappa^{++}$but $2^{\kappa}$ is as large as desired.

All these results only concern regular cardinals and their successors, and leave open the question for singular cardinals and their successors. Positive results analogous to the one just mentioned by Džamonja and Shelah were obtained by Džamonja and Shelah, again, in [14], for the case where $\kappa$ is a singular cardinal of countable cofinality, and by Cummings, Džamonja, Magidor, Morgan and Shelah in [9], for the case where $\kappa$ is a singular cardinal of arbitrary cofinality. The most general of their results can be stated as follows:

Theorem 4 /Cummings et al. [9]/ If $\kappa$ is a supercompact cardinal, $\lambda<\kappa$ is a regular cardinal and $\Theta$ is a cardinal with $\operatorname{cf}(\Theta) \geq \kappa^{++}$and $\kappa^{+3} \leq \Theta$, then there is a cardinal preserving forcing extension in which $\operatorname{cf}(\kappa)=\lambda, 2^{\kappa}=2^{\kappa^{+}}=\Theta$ and in which there is a universal family of graphs on $\kappa^{+}$of size $\kappa^{++}$.

Further recent results of Shelah (private communication) indicate that the universality number in the above model should be exactly $\kappa^{++}$. These results concern successors of singular cardinals, which themselves are, of course, regular. The situation for singular cardinals themselves is different; in particular, no forcing notion can operate on them. We do not have any general results about graphs on such cardinals, but here is a result showing that in specific classes of graphs, the existence of a universal element at singulars is simply ruled out by the axioms of ZF (not even the full ZFC is needed): 
Theorem 5 [Džamonja [13]] (ZF) Suppose that $\kappa$ is a cardinal of cofinality $\omega$. Then, for any $\lambda \geq \kappa$ in $Z F$, there is no universal element in the class of graphs of size $\lambda$ that omit a clique of size $\kappa$, under graph homomorphisms, or the weak or the strong embeddings.

This survey of the graph universality problem shows in a specific example the phenomenon of the change in the combinatorial behaviour between the three kinds of cardinals: successors of regulars, successors of singulars and, finally, singular cardinals. At successors of regulars combinatorics is very independent of ZFC, so that simple forcing, without use of large cardinals, allows us to move into universes of set theory which have very distinct behaviours. At the successor of a singular cardinal, we can move from $L$-like universes only if we use large cardinals (as we know by Jensen's Covering, mentioned above), and this shows up in combinatorics in the necessity to use both large cardinals and forcing to obtain independence results. This independence is in fact limited (as in the example of Shelah's pcf theorem quoted above). Finally, at singular cardinals, combinatorics tends to be completely determined by ZFC, or even by ZF, as in the example of Theorem 5 .

In connection with this theorem, it is interesting to note that in the absence of the Axiom of Choice, it is possible that every uncountable cardinal is singular of countable cofinality. To be exact, Gitik proved in [18] that from the consistency of ZFC and arbitrarily large strongly compact cardinals, it is possible to construct a model of ZF in which all cardinals have countable cofinality. Therefore, if one is happy to work with ZF only, then one has the choice to move to a model in which only singular cardinals exist and they only have countable cofinality. In such a model, combinatorics becomes easy and determined by the axioms, at least in the context of the questions that have been studied, such as the graph universality problem.

\section{Philosophical Remaks}

Mathematical platonism is often presented as the thesis that mathematical objects exist independently of any sort of human (cognitive, and/or epistemic) activity, and it is taken to work harmoniously with a realistic semantic view, according to which all we can say in mathematics (i.e. by using a mathematical language) is either true or false, to the effect that all that has been (unquestionably) proved is true, but not all that is true has been (unquestionably) proved or can be proved (because of various forms of incompleteness of most mathematical theories).

Both claims are, however, quite difficult to support and are, in fact, very often supported only by the convenience of their consequences, or, better, by the convenient simplicity of the account of mathematics they suggest, and because they provide a simple explanation of the feeling most mathematicians (possibly all) have that something external to them resists their intuitions, ideas, programs, and conjectures, to the effect that all that they can frame by their thoughts or their imagination must have, as it were, an external, independent approval, before having its place among mathematical achievements. Hence, an interesting philosophical question is whether there can be weaker claims that have similarly convenient consequences and that can be more easily positively supported, either by evidence coming from mathematical practice, or by more satisfactory metaphysical assumptions, or, better, by both.

It is our opinion that such claims can reasonably be formulated. In short, they are the following: i) there are ways for us to have epistemic de re access to mathematical objects; ii) we are able to prove truths about them, though others are still not proved or are unprovable within our 
most convenient theories (which are supposed to deal with these objects). Claim $(i)$ means that there are ways for us to fix intellectual contents which are suitably conceived as individuals that mathematics is dealing with, in such a way that we can afterwards (that is, after having fixed them) ascribe properties and relations to these individuals. Claim (ii) means that some of our ascriptions of property and relations to these individuals result in truths, in the sense that they somehow comply with the content we have afterwards fixed, and, among them, some can be, and in many cases have been, provably established, though others are still not so or cannot be so within the relevant theories.

The phrase ' $d e$ re' in claim $(i)$ belongs to the philosophical lexicon. It is currently used in opposition to 'de dicto' to point out a distinction concerning propositional attitudes, typically belief (or knowledge). Believing that something is $P$ can mean believing either that there is at least one thing that is $P$ or that some specific thing is $P$. In the former case the belief is de dicto; in the latter de re. If the relevant thing is $t$, a suitable way to unambiguously describe the second belief is saying that of $t$, it is believed that it is $P$. This makes clear that the subject of a de re propositional attitude is to be identified independently from ascribing to it what the relevant proposition ascribes to it. Hence, its being $P$ cannot be part of what makes it what it is. This is not enough, however, since for the attitude to be de re, the identification has to be stable under its possible variations. If Mirna believes that $t$ is the only thing that is $Q$, her believing of $t$ that it is $P$ is the same as her believing it of the $Q$. But Marco can believe that the only thing that is $Q$ is $s$ (distinct from $t$ ). So his believing of the $Q$ that it is $P$ is quite distinct from Mirna's belief that it is so. Hence neither beliefs are de re. This makes clear that the identification of the subject of a de re attitude is to be independent of the attitude itself or, even, of any sort of attitude (since different attitudes can compose each other's). This is why the most straightforward examples of de re attitudes concern empirical objects ostensively, or pre-conceptually identified in one way or another.

This has not prevented philosophers from appealing to the de re vs. de dicto distinction in relation to mathematics. In particular, a rich discussion has concerned the possibility of using appropriate sorts of numerals for directly referring to natural numbers while having a de re attitude towards them. Diana Ackerman has considered that "the existence of [natural] numbers is a necessary condition for anyone's having de re propositional attitudes toward them" ([1], p. 145). Granted their existence, Tyler Burge has wondered whether we can have "a striking relation to [...] [a natural number] that goes beyond merely conceiving of it or forming a concept that represents it", and answered that this is so for small such numbers, since "the capacity to represent [...][them] is associated with a perceptual capacity for immediate perceptual application in counting" ([6], pp. 70-71). Saul Kripke has gone far beyond this, by suggesting a way to conceive natural numbers that makes decimal numerals apt to "reveal[...] their structure" ([39], p. 164; [29]). For him, natural numbers smaller than 10 are the classes of all $n$-uples $(n=0,1, \ldots, 9)$, while those greater than 9 are nothing but finite sequences of those smaller than 10. This makes decimal numerals, or, at least, short enough ones, work as "buckstoppers" (i.e. they are such that it would be nonsensical asking which number is that denoted by one of them, in opposition to terms like 'the smallest perfect number', denoting the natural number whose buckstopper is 'six'), and so allow direct reference to them. By dismissing such a compositional conception of natural numbers, Jan Heylen ([25] and Stewart Shapiro ([38]) have respectively submitted that Peano numerals (the numerals of the form ' 0 '..', written using only the primitive symbols for zero and the successor relation in the language of Peano Arithmetic) and unary numerals (mere sequence of $n$ strokes used 
to denote the positive natural number $n$ ) provide canonical notations allowing de re knowledge of natural numbers. Finally, Jody Azzouni ([3]) has argued that the existence of natural numbers is not required for having "de re thought" about them, since such a thought can be "empty".

Our use of 'de re' in claim $(i)$ differs from all these uses in that the de re vs. de dicto distinction has a much more fundamental application in our account of mathematics. Far from merely concerning our way of denoting natural numbers so to identify them in such a way to make de re propositional attitudes towards them possible, granted their existence, or our de re thought about them empty, granted their nonexistence, it concerns our way of fixing mathematical objects so as to confer existence to them. In our view these objects are, indeed, nothing but contents of (intentional) though, whose existence just depends on the way they are fixed. Here is how we see the matter.

There are many ways of fixing intellectual contents, which, in appropriate contexts, are (or can be) suitably conceived as individuals. A liberal jargon can refer to these contents as abstract objects. If this jargon is adopted, the claim that mathematics deals with abstract objects becomes quite trivial, and can neither be taken as distinctive of a platonist attitude, nor can provide any characterisation of mathematics among other intellectual enterprises. In a much more restrictive jargon, for something (i.e. the putative reference of a term or description) to count as an object, it has to exist. Under this jargon, the claim that mathematics deals with abstract objects becomes much more demanding, overall if it is either required that these objects are self-standing or mind-independent, or if it is supposed that nothing can acquire existence because of any sort of intellectual (intentional) act. The problem, then, with this claim is that it becomes quite difficult to understand what 'to exist' can mean if referred to abstract contents. What we suggest is reserving the term 'abstract object' to intellectual contents suitably conceived as individuals and so fixed, in an appropriate context, so as to admit de re epistemic access, this being conceived, in turn, as the apprehension of them making de re attitudes towards them possible. We submit that, once this is granted, the claim that mathematics deals with abstract objects becomes both strong enough and distinctive, so as to provide the ground for an appropriate account of mathematics.

Mathematics traditionally admits different modalities for fixing intellectual contents. The French philosopher Jean-Michel Salanskis ([34], [35]) suggested to distinguish two basic ways of doing it: constructively and correlatively.

The former way has a more limited application, but can be taken, in a sense, as more fundamental. Peano's numerals can, for instance, be quite simply fixed constructively by stating that: $i$ ) the sign ' 0 ' is a Peano's numeral; $i i$ ) if the sign ' $\sigma$ ' is such a numeral, then the sign ' $\sigma$ ' is such a numeral, too; iii) nothing else is such a numeral. Similarly, unary numerals can be constructively fixed by stating that: $i$ ) the sign ' $\mid$ ' is such a unary numeral; $i i$ ) if the sign ' $\sigma$ ' is such a numeral,

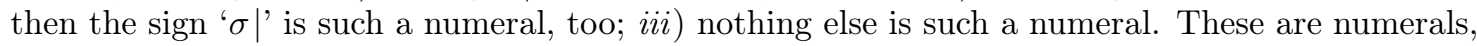
not numbers, however. And is clearly unsuitable to use the same pattern to define natural numbers. Suppose it were stated that: i) 0 is a natural number; ii) if $\sigma$ is such a number, then $\sigma^{\prime}$ is such a number; iii) nothing else is such a number. It would have not been established yet that there is no natural number $n$ such that $0=n^{\prime}$, or $n=n^{\prime}$. To warrant that this is so, it would still be necessary to impose appropriate conditions to the successor function $-^{\prime}$, which cannot be done constructively. To overcome the difficulty, one could have recourse to a trick: stating the natural numbers are the items that Peano numerals denote, or positive such numbers the items that unary numerals denote, in such a way that distinct such numerals denote distinct such numbers. This would make Peano's numerals directly display the structure of natural numbers, and 
unary ones that of positive natural numbers, so providing a canonical notation for these numbers allowing direct reference to them, in agreement to Heylen's and Shapiro's proposals. But this would be dependent on the informal notion of denotation. Supposing that we have the necessary resources for handling this notion without ambiguity, this would allow us to fix natural numbers almost constructively. Once this is done, one could look at these numbers as such, and try to disclose properties they have and relations they bear to each other's. Making it in agreement with mathematical requirements of rigor asks both for further definitions and the fixation of inferential constraints or rules, typically of an appropriate codified, if not formal, language. What is relevant for illustrating our point, is, however, not this, but rather that that we can do both things in such a way to keep the reference steady to the contents previously fixed as just said: it is on them that we define the relevant properties and relations; and it is to speak of them that we establish the appropriate inferential constraints, and fashion (or adopt) the appropriate language, which allows us to say of them, or some of them, that they are so and so. This should give a rough idea of the intellectual phenomenon we want to focus on by speaking of de re epistemic access.

More importantly, we could observe that once appropriate intellectual contents are fixed constructively, one can also try to capture them correlatively, that is, through an axiomatic implicit definition. This can be done somehow informally, or by immersing the definition within a formal system affording both the appropriate language and the appropriate inference rules (or, possibly, allowing to state these rules). In the case of natural numbers, we can, for instance, define them, through Peano axioms, within an appropriate system of predicate logic, and we could conceive of doing that with the purpose of characterizing correctively the same contents previously fixed constructively, so as that each of them provide the reference for a singular term appropriately introduced within the adopted language, and that they provide, when taken all together, the domain of variation and quantification of the individual variables involved in the definition.

The predicate system adopted can be both first- or higher-, typically second-, order. There is, however, a well-known difference among the two cases: while Peano second-order arithmetic (or PA2, for short) is categoric (with respect to the subjacent set theory), by a modern reformulation of Dedekind's argument ([10]), Peano first-order arithmetic (or PA1, for short) is not, by an immediate consequence of the Löwenheim-Skolem's theorem ([7]). This suggests that the verb 'to capture' is not to be understood in the same way in both cases. In the second-order case, it means that the relevant axioms determine a single structure (up to isomorphism), whose elements are intended to be the natural numbers, identified with the same objects previously fixed constructively. In the first-order case, it means that these axioms describe a class of non-isomorphic structures, all of which include individuals that behave, with respect to each other's, in the same way as the elements of this structure do, and that we can then intend, again, as the same objects previously fixed constructively.

Both in the usual platonist tongue, and in our amended one, we could say that the limited expressive power of a first-order language makes it impossible to univocally describe the natural numbers by means of such a language: to do it, a second-order language is needed (and it suffices). Still, the verb 'to describe' should be understood differently in the two cases: while in the former case it implies that that these numbers are self-standing objects that are there as such, independently of any intellectual achievement, in the latter case, it merely implies that these objects have been previously fixed. Hence, if no previous definition were admitted or considered, the verb 'to fix' should be used instead. What should, then, be said is that the limited expressive power of a first-order language makes it impossible to univocally fix the natural numbers by means of such a 
language. (Of course, the relativisation of the categoricity of PA2 to a given model of set-theory makes the usual platonist tongue appropriate only insofar as it is admitted that this model reflects the reality of the world of mathematical objects, which, in presence of the strong non-categoricity of ZFC requires a further act of faith. But on this, later.)

The difference between and first- and the second-order case is not limited to this, however. Another relevant fact is that the language of PA1 is forced to include, together with the primitive constants used to designate the number zero and the successor relation, also two other primitive constants used to designate addition and multiplication. (Though versions of PA1 often adopt a language including a further primitive constant used to designate the order relation, this can be easily defined in terms of addition by, then, reducing the number of axioms, albeit increasing the syntactical complexity of some proofs.) The only primitive constants which are required to be included in the language of PA2 are, instead, those used to designate the number zero and the successor relation: addition and multiplication (as well as order), can be recursively defined in terms of zero and successor. Hence, whereas Peano second-order axioms (implicitly) define a structure $\left\langle\mathbf{N},{ }^{\prime}\right\rangle$ Peano first-order axioms define uncountably many distinct structures $\left\langle\mathbf{N},{ }^{\prime},+, \times\right\rangle$. It remains the fact, nevertheless, that the former structure is reflected within any one of the latter ones. Hence, if we admit that the axioms of PA2 capture or fix a domain of objects in an appropriate way, there is room to say that PA1 is studying these same objects by weaker logical means, by identifying them as the common elements of uncountably many possible structures $\left\langle\mathbf{N},{ }^{\prime},+, \times\right\rangle$, though being unable to provide an univocal characterisation of them.

This should clarify a little better what having epistemic de re access to mathematical objects could mean: one could argue that, once natural numbers are captured or fixed by the axioms of PA2 as the elements of $\left\langle\mathbf{N},{ }^{\prime}\right\rangle$, one can, again, look at them as such and try to disclose their properties and relations, so as to recover the same property or relation already ascribed to them, and possibly more. This can be done in different ways. By staying within PA2, one can, for example, besides proving the relevant theorems statable in its primitive language, also enrich this language by means of appropriate explicit definitions, so as to introduce appropriate constants - as those designating addition multiplication and order - to be used in the relevant proofs. By leaving this theory, one can also try to describe them by using a weaker language, such as a first-order one, and be, then, forced to implicitly define addition and multiplication in them by appropriate axioms, though being unable to reach an univocal description. Other ways for studying these numbers are, of course, at hand. But, for our present purpose, we can confine ourselves to observe that in this latter case (as in many other ones), what we are doing may be appropriately accounted for by saying that, of these very numbers, we claim (by using the relevant first-order language) that they are so and so, or, better, that they form a structure $\left\langle\mathbf{N},{ }^{\prime},+, \times\right\rangle$.

There is a quite natural objection one could address to these views. One could remember that, as any other second-order theory, PA2 is syntactically incomplete, to the effect that some statements that are either true or false in its unique model are neither provable nor disprovable in it, and there is, then, no way (or at least no mathematically appropriate way) for us to know whether they are true or false. Hence, one could argue, whatever a de re access to natural numbers, as defined by PA2, might be, it cannot be, properly speaking, an epistemic access, since there are not only things about these numbers that we do not know, but also things that we cannot know. We think this objection misplaced, since something analogous also occurs for genuine empirical objects. Take the chair you sit on (if any): there are many properties that we suppose (at least from a realist perspective) that it does or does not have, about which even our best theories and 
the information we are in place to obtain are insufficient to make a decision. This should not imply, it seems to us, that you have no knowledge of that chair. Of course, we could always change our theories or improve them if we considered that deciding some questions that we know to be undecidable within them is relevant. In the same way, if we were considering (or discovering) that there are some relevant statements about natural numbers which are provably undecidable in PA2, we could try to add axioms to the effect of provably deciding these statements. But allowing this possibility does not imply that we do not have de re epistemic access to these numbers as fixed by PA2, while working on them either within or outside it. All that is required for it is that there is a suitable sense in which we can say that on these numbers (as independently fixed) we can define some properties or relations within this theory, or of these numbers we can claim this or that outside the theory.

Something similar to what happens with PA2 also happens with Frege arithmetic (or FA, for short), namely full (dyadic) second-order logic plus Hume's Principle (see Wright [49] or [4], especially section II). The role played by natural numbers in the former case is played by the cardinal ones (understood as numbers of concepts) in the latter case. Once a particular cardinal number, typically the number of an (or the) empty concept is identified with 0 , and an appropriate functional and injective relation is defined on these numbers so as to play the role of the successor relation, one can select the natural numbers among the cardinal ones, as being 0 together with all its successors. One can then capture or fix the natural numbers without appealing to addition and multiplication on them (and no more on order, at least explicitly). But now there is even more: these numbers can be captured or fixed by selecting them among items which are fixed, in turn, by appealing neither to a designated item like 0 , nor to a certain dyadic relation, like the successor relation. Of the cardinal numbers, one could, then, say, that some of them are the natural ones and can be studied as such with other appropriate means.

It is easy to see that, as opposed to PA2, FA is not categoric (with respect to the subjacent set theory). This merely depends on the presence in some of its models of objects other than cardinal numbers, which can be absent from others. Still, FA interprets PA2 (this is generally known as Frege's theorem: see [22], for example), and a result of relative categoricity can also be proved for FA ([47], prop. 14; [48], pp. 573-574): any two models of it restricted to the range of the number-of operator are isomorphic (with respect to the subjacent set theory). This migh $t$ make one think that a form of categoricity (with respect to the subjacent set theory) is essential for allowing de re epistemic access to mathematical objects, i.e. that the only intellectual contents suitably conceived as mathematical objects that we can take to have de re epistemic access to are those fixed within a theory endowed with an appropriate form of categoricity (with respect to the subjacent set theory).

This is not what we want to argue for, however. The previous example of the constructive definition of positive natural numbers should already make it clear. Another, quite simple example is the following: when we define the property of being a prime number within PA1, we do it on the natural numbers in such a way that we can say that on these numbers we define this property; if the definition is omitted, many usual theorems of PA1 can no longer be proved, of course, but this does not change anything to many other theorems still concerned with natural numbers as defined within this theory. These two examples are different from each other, and both different from that given by the access to natural numbers as defined within PA2. That provided by the definition of prime numbers within PA1 is only an example of de re epistemic access internal to a given theory, which reduces, in fact, to nothing more than the possibility of performing an 
explicit definition within this very theory. Claiming that we have de re epistemic access to natural numbers as defined constructively, or to these very numbers as defined correlatively within PA2, when we try to study them in a different context, is quite a different story. Still, there is something similar in the three cases, and this is just what we are interested in underlining here: it is a sort of (relative) stability of intellectual contents counting as mathematical objects, a stability that is made possible by the way these contents are fixed. We do not want to venture here in the (possibly hopeless) tentative of classification of forms of de re epistemic access. Still, it seems clear to us that the phenomenon admits differences: both the stability depending on a constructive, or, more generally, informal definition, and that depending on a categorical implicit formal definition are extra-theoretic; the former is strictly intentional, as it were, the latter semantic; that depending on explicit definitions within non-categoric theories is merely syntactic (and, then, intra-theroretic) or restricted, at least, to an informally identified intended model. But the notion of independent existence of mathematical objects, which usual platonism is concerned with, is imprecise enough to make it possible to hope that all these different sorts of stability can provide an appropriate (metaphysically weak) replacement of it in many cases in which platonists use it in their accounts of mathematics.

$$
\# \# \#
$$

But, let it be as it may. The question here is different: what does all this have to do with ZFC, and the results mentioned in $\S \S 2$ and 3 , above?

On the one side, it is clear not only that the categoricity of PA2 and FA is relative to the (inevitably arbitrary) choice of a model of set-theory, and, then, typically, of ZFC, but also that what has been said about PA1, PA2 and FA has a chance to be clear only if set-theory provides us with a clarification of the relevant crucial notions. This is, however, not enough for concluding that whatever philosophical position we could take on natural numbers, and other mathematical objects along the lines suggested above, is necessarily dependent on a preventive account of ZFC. On the one side, we do not need all the expressive and deductive power of ZFC, and a fortiori of whatsoever acceptable extension of it, to make the relevant notions clear. On the other side, it is exactly the high un-categoricity of ZFC that invites us to reason with respect to finite numbers under the supposition that a model of the subjacent set-theory has been chosen, or, even, independently of the preventive assumption that these numbers are sets.

This suggests taking ZFC as an independent mathematical theory - one, by the way, powerful enough to be used (among other things) for studying from the outside the structures formed by the natural numbers, as well as by other mathematical objects, as objects we have a de re epistemic access to independently of (the whole of) it. One could then ask whether some sort of de re epistemic access to pure sets (conceived as sui generis objects implicitly defined by ZFC) is possible or conceivable. The high un-categoricity of ZFC seems to suggest a negative answer. Because it looks like neither this theory as such, nor any suitable extension of it (with the only exception, possibly, of ZFC $+{ }^{\prime} V=L$ ', if this might be taken to be a suitable theory, at all) can provide a way to fix pure sets in any appropriate way for allowing de re (semantic) epistemic access to them. Upon further reflection, the case can appear, however, not to be so desperate as it seems to be at first glance, and the results mentioned above help us in seeing why this is so.

To begin with, one might wonder whether, in analogy to what we have said concerning PA1 and PA2, ZFC could not be taken as studying pure sets as the objects previously fixed in a quasi- 
categorical way by ZF2, just like PA1 might be taken to do with the natural numbers as (captured or) fixed by PA2.

The problem with this suggestion is that the relations between ZFC and ZF2 are not as illuminating as those between PA1 and PA2. For example, if we fix a level of the cumulative hierarchy of sets, say $V_{\alpha}$, then the second-order theory of $V_{\alpha}$ is simply the first-order theory of $\mathcal{P}\left(V_{\alpha}\right)=V_{\alpha+1}$, hence passing to the second-order does not seem like it has achieved much. However, it is true that formulating ZF in the full second-order logic so as getting ZF2, one achieves what is known as quasi-categoricity. The proof is basically contained in Zermelo [50]. We can describe the situation in more detail although informally, as follows.

What Zermelo proved for ZF2 is that for any strongly inaccessible cardinal $v$ which is supposed to exist, there is a single model (up to isomorphism) of ZF2 provided by the structure $\left\langle V_{v}, \in\right\rangle$. It follows that all theories ZF2 + 'there are exactly $n$ strongly inaccessible cardinal' $(n=0,1,2, \ldots)$, or ZF $2_{n}$, for short, are fully categorical, giving that ZF2 has, modulo isomorphism, as many (distinct) models as there are strongly inaccessible cardinals (recall that $V_{v}$ can only include strongly inaccessible cardinals smaller than $v$ ). Of course, in any of these models any statement of the language of ZF2 is either true or false (according to the Tarski's semantic). But, because of the proof-theoretical incompletess of the second-order logic, and, then, of any second-order theory, it is not necessarily decidable. As noted below, this is so also for PA2. The difference is that in these extensions of ZF2, the undecidable statements include some with a clear and unanimously perfectly recognized mathematical significance, namely $\mathrm{CH}$ and $\mathrm{GCH}$.

Now, while the problem of deciding GCH (for cardinals greater than $2^{\aleph_{0}}$ ) can be seen as intrinsically internal to set theory (both to ZFC and ZF2), this is not so for CG. For, if we admit that there are (necessarily not-constructive) ways to fix real numbers, so as to allow us to have de re epistemic access to them (for example within PA2, as originally suggested by Hilbert and Bernays ([24], supplement IV), the problem of deciding $\mathrm{CH}$ can be seen as the question of answering the very natural question of how many are such numbers, a question which should, then, be seen as having a definite answer outside set theory (both ZFC and ZF2). The difference is, then, relevant, also from the point of view we are delineating.

Usually, a model $V_{M}$ of ZFC is diagrammatically represented this way:

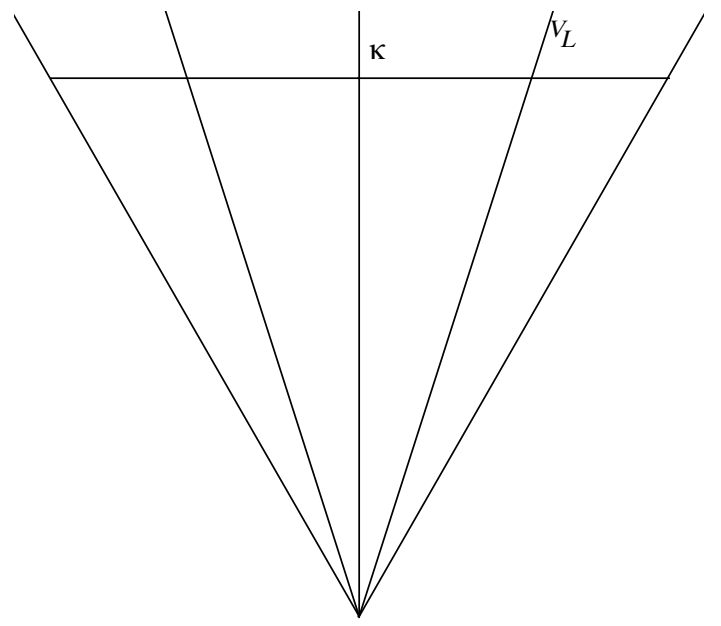


where $V_{L}$ is the model of ZFC + ' $V=L$ ', and the external triangle can coincide with the internal one (which happens if ' $V=L$ ' is true in the model), but not go up to become internal. However, insofar as nothing requires that a model of ZFC have a uniform hierarchic shape, and no significant feature of it is represented by the symmetry of the diagram, we submit that a better representation is the following

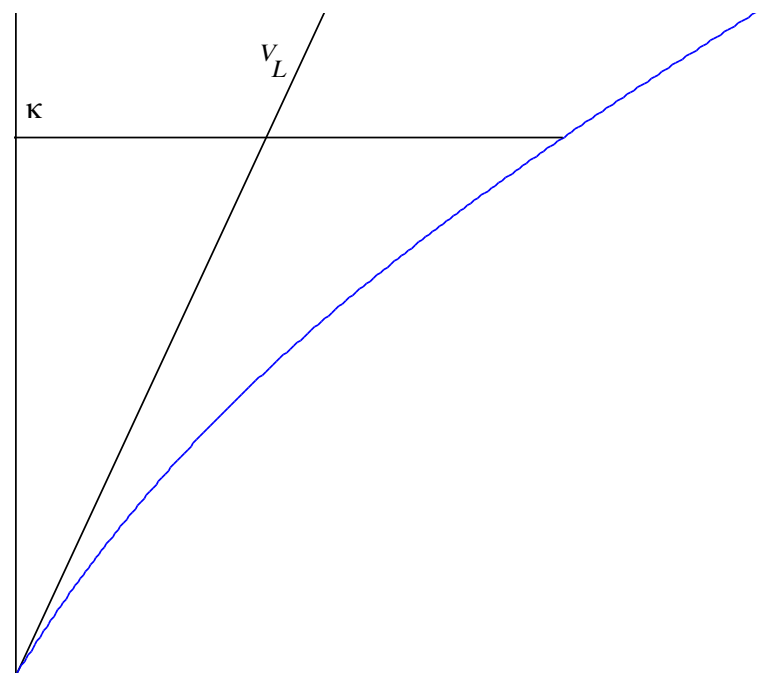

where all that is required of the external curve, call it ' $\mathcal{C}$ ', for short, is that it is everywhere increasing (with respect to the line of cardinals, taken as axe) and external or coincident to the internal half straight-line. If this picture is adopted, a model of ZF2 could be depicted in the same way, with the specification that the external curve is univocally determined by the choice of a strongly inaccessible cardinal $v$, or by the supposition that there are exactly $n$ such cardinals, which leads to our calling it ' $\mathcal{C}_{v}$ ' or ' $\mathcal{C}_{n}$ '.

One could, then, advance that (the axioms of) ZF2 plus the choice of a strongly inaccessible cardinal, or (those of) $\mathrm{ZF} 2_{n}$ allow to univocally fix a domain of sui generis objects - call it 'the $v$-sets' or 'the $n$-sets' - and that ZFC is studying these very objects with weaker logical means as elements of uncountably many possible structures, being unable to provide an univocal characterisation of them.

This suggests that ZF2, plus the choice of a strongly inaccessible cardinal, or ZF $2_{n}$ provide domains of objects we can have a de re access to, in the same way as this happens for PA2, that is, not only internally, and so providing a sort of syntactic stability, but also externally, so as to provide a sort of semantic stability: one could argue that, once pure sets are fixed by the relevant (second-order) axioms, one can look at them as such and try to tell (both using a firstor a second-order language) the properties they have or the relations they bear to each other's. Of them, we claim that they form a structure that $\mathrm{ZF}(\mathrm{C})$ and all its usual (first-order) extensions try to describe, though being unable to univocally identify.

Still, the relativisation to the choice of a strongly inaccessible cardinal or the admission of the supplementary axiom 'there are exactly $n$ strongly inaccessible cardinals' make the situation much less satisfactory than the one concerned with Peano (first- and second-order) arithmetic: taken as 
such, ZF2 is not only proof-theoretically incomplete; it is also unable to univocally fix the relevant objects.

This relativisation or admission do not prevent us from ascribing, however, to ZF2 a form of categoricity, since from Zermelo's result "it also follows that every set-theoretical question involving only sets of accessible rank is answerable in ZF2", and, then, in particular, that "all propositions of set theory about sets of reals which are independent of ZFC", among which there is CH, are either true or false in any of its model, though no proof could allow us to establish whether the former or the latter obtains ([26], p. 790). This might be taken as very good news. But a strong objection is possible: it is possible to argue that the truth or falsity of $\mathrm{CH}$ in any model of ZF2 does not depend on the very axioms of this theory, but on the consequence relation which is determined by the use of second-order logic and the standard (or full) interpretation of it, or, in other terms, that what makes $\mathrm{CH}$ true or false there is not what the axioms of ZF2 genuinely say about sets, but their using second-order variables, semantically interpreted as sets of $n$-tuples on the fist-order domain. Clearly, this would make second-order logic so interpreted "inadequate for axiomatizing set theory" (see [26], pp. 782 and 790-793, for details).

We do not want enter such a delicate question here. We merely observe that the mathematical results we have expounded above show that there is no need to go second- order to get a limited form of quasi-categoricity. Since these results suggest that ZFC has already (and alone, that is, without any need to appeal to any supplementary axiom) the resources for fixing some of its objects in a better way than it is usually thought. Namely, if we are happy to work at a singular cardinal then much of the combinatorics is determined by what happens at the regular cardinals below, even to the point of fixing the cardinal arithmetic (see Shelah's theorem 1 quoted above). In some cases, we do not even need to know what happens at the regular cardinals below (see theorem 5). And if we are happy to be in a world with no Axiom of Choice, we can even imagine that all cardinals are singular, as in the Gitik's model and hence much of the cardinal combinatorics is completely determined by ZF.

Let us look back to the second of the previous figures and suppose that $\kappa$ is a singular cardinal. What these results suggest is this: if the values of the ordinates of $\mathcal{C}$ are fixed for all regular cardinals $\lambda$ smaller than $\kappa$, i.e. if a single model of ZFC is chosen relatively to all these regular cardinals, then the value of the ordinate of $\mathcal{C}$ for $\kappa$ is strongly constrained, in the sense that this value can only belong to a determined set (a set, not a class) of values. In other terms, things seem to happen as if the shape of a model of ZFC for the regular smaller than $\kappa$ strongly conditions the shape of the possible models at $\kappa$.

These results could be understood as saying that the non-categoricity of ZFC is, in fact, not as strong as it appears. Even within first-order, the behavior of the universe of sets is fixed enough at singular cardinals to give us some sort of external and semantic de re epistemic access to them and their power sets. In particular, once we have given to us all sets of size $<\kappa$ and all their power sets, our choices for $\kappa$ are quite limited. This offers an image of the universe of sets in which a strong lack of uniuvocality only concerns successor cardinals or uncountable regular limit cardinals, if any (remember that the existence of uncountable regular limit cardinals is unprovable in ZFC). One could say that, at singular limits, ZFC already exhibits a form of categoricity, or, better, that it does it asymptotically, since the ratio of singular cardinals over all cardinals tends to 1 as the cardinals grow. And at the price of working only in ZF we can even imagine to be in the model of Gitik, in which every uncountable cardinal is a singular limit.

Under a realist semantic perspective, according to which all we could say about the universe of 
sets is either true or false, one could say that this shows that, though ZFC is unable to prove the full truth about this universe, it provably provides an asymptotic description where the singular cardinals are the limits of the asymptotes. This also suggests, however, an alternative and more sober picture, which is what we submit: though there is no sensible way to say what is true or false about the universe of sets, unless truth and falsity are merely conceived as provable truth and falsity, ZFC provides an asymptotically univocal image of the universe of sets around the singular cardinals: the image of a universe to which we can have an external semantic de re epistemic access.

Acknowledgement 6 The first author gratefully acknowledges the help of EPSRC through the grant EP/I00498, Leverhulme Trust through research Fellowship 2014-2015 and l'Institut d'Histoire et de Philosophie des Sciences et des Techniques, Université Paris 1, where she is an Associate Member. The second acknowledges the support of ANR through the project ObMathRe. The authors are grateful to Walter Carnielli for his instructive comments on a preliminary version of the manuscript, and to Marianna Antonutti-Marfori, Drew Moshier and Rachael Schiel for valuable suggestions.

\section{References}

[1] Diana Ackerman. De re propositional attitudes toward integers. The Southwestern Journal of Philosophy, 9(2):145-153, 1978.

[2] Wilhelm Ackermann. Die Widerspruchsfreiheit der allgemeinen Mengenlehre. Math. Ann., 114(1):305-315, 1937.

[3] Jody Azzouni Empty de re attitudes about numbers Philosophia Mathematica, ser. III, 17(2):163-188, 2009.

[4] George Boolos. Logic, logic and logic. Harvard University Press, October 1999.

[5] Lev Bukovský. The continuum problem and powers of alephs. Comment. Math. Univ. Carolinae, 6:181-197, 1965.

[6] Tyler Burge Postscipt to "Belief De Re". In Tyler Burge, Foundations of Mind. Philosophical Essays, Volume 2, pages 65-81. Clarendon Press, Oxford, New York, etc., 2007.

[7] Chen Chung Chang and H. Jeremy Keisler. Model Theory. North Holland, Amsterdam, third edition, 1990.

[8] Paul Cohen. The independence of the continuum hypothesis. Proc. Nat. Acad. Sci. USA, 50(6):1143-1148, 1963.

[9] James Cummings, Mirna Džamonja, Menachem Magidor, Charles Morgan, and Saharon Shelah. A framework for forcing constructions at successors of singular cardinals. To appear in TAMS (submitted in 2013), 201x.

[10] Richard Dedekind. Was sind und was sollen die Zahlen? Braunschweig, 1888. 
[11] Keith I. Devlin and R. B. Jensen. Marginalia to a theorem of Silver. In $\vDash I S I L C$ Logic Conference (Proc. Internat. Summer Inst. and Logic Colloq., Kiel, 1974), pages 115-142. Lecture Notes in Math., Vol. 499. Springer, Berlin, 1975.

[12] Mirna Džamonja. The singular world of singular cardinals. In Åsa Hirvonen, Juha Kontinen, Roman Kossak, and Andrés Villaveces, editors, Logic Without Borders: Essays on Set Theory, Model Theory, Philosophical Logic and Philosophy of Mathematics, pages 139-146, Boston, 2015. De Gryter.

[13] Mirna Džamonja. ZFC combinatorics at singular cardinals. Sarajevo Journal of Mathematics, 12(25):151-154, 2016. to appear.

[14] Mirna Džamonja and Saharon Shelah. Universal graphs at the successor of a singular cardinal. J.Symbol. Logic, 68:366-387, 2003.

[15] Mirna Džamonja and Saharon Shelah. On the existence of universal models. Arch. Math. Logic, 43(7):901-936, 2004.

[16] William B. Easton. Powers of regular cardinals. Ann. Math. Logic, 1:139-178, 1970.

[17] Fred Galvin and András Hajnal. Inequalities for cardinal powers. Ann. of Math. (2), 101:491498, 1975.

[18] Moti Gitik. All uncountable cardinals can be singular. Israel J. Math., 35(1-2):61-88, 1980.

[19] Kurt Gödel. Über formal unentscheidbare Säztze der Principia Mathematica und verwandter Systeme, I. Monatshefte für Mathematik und Physik, 38:173-198, 1931.

[20] Kurt Gödel. The Consistency of the Continuum-Hypothesis. Princeton University Press, Princeton, 1997.

[21] Stephen H. Hechler. Powers of singular cardinals and a strong form of the negation of the generalized continuum hypothesis. Z. Math. Logik Grundlagen Math., 19:83-84, 1973.

[22] Richard G Heck. Frege's Theorem. Clarendon Press, Oxford, 2011.

[23] Pavol Hell and Jaroslav Nešetřil. Graphs and homomorphisms, volume 28 of Oxford Lecture Series in Mathematics and its Applications. Oxford University Press, Oxford, 2004.

[24] David Hilbert and Paul Bernays. Grundlagen der Mathematik. Springer, Berlin, Heidelberg, New York, 1934-1939 (2 vols.). Second edition: 1968-1970.

[25] Jan Heylen The epistemic significance of numerals Synthese, Published online on September $6^{\text {th }}, 2014$. https://doi.org/10.1007/s11229-014-0542-y

[26] Ignacio Jané. Higher-order logic reconsidered. In [37], pp. 781-808.

[27] Thomas Jech. Set Theory. Springer-Verlag, Berlin Heidelberg, 3rd millenium edition, 2003. 
[28] Menachem Kojman. Singular Cardinals: from Hausdorff's gaps to Shelah's pcf theory. In Dov M. Gabbay, Akihiro Kanamori, and John Woods, editors, Sets and Extensions in the Twentieth Century, volume 6 of Handbook of the History of Logic, pages 509-558. Elsevier, 2011.

[29] Saul Kripke. Whitehead Lectures. Umpupished transcription of Kripke's lectures delivered at Harvard University on May $5^{t h}-6^{\text {th }}, 1992$.

[30] Menachem Kojman and Saharon Shelah. Nonexistence of universal orders in many cardinals. J. Symbolic Logic, 57(3):875-891, 1992.

[31] Menachem Magidor. On the singular cardinals problem. I. Israel J. Math., 28(1-2):1-31, 1977.

[32] A. Mekler. Universal structures in power $\aleph_{1}$. Journal of Symbolic Logic, 55(2):466-477, 1990.

[33] Richard Rado. Universal graphs and universal functions. Acta Arith., 9:331-340, 1964.

[34] Jean-Michel Salanskis. L’heméneutique formelle. Éditions du CNRS, Paris, 1991.

[35] Jean-Michel Salanskis. Philosphie des mathématiques. Vrin, Paris, 2008.

[36] Dana Scott. Measurable cardinals and constructible sets. Bull. Acad. Polon. Sci. Sér. Sci. Math. Astronom. Phys., 9:521-524, 1961.

[37] Stewart Shapiro. The Oxford Handbook of Philosophy of Mathematics and Logic. Oxford Univ. Press, Oxford, New York, etc., 2005.

[38] Stewart Shapiro Computing with numbers and other non-syntactic things: De re knowledge of abstract Philosophia Mathematica, ser. III, 25(2):268-281, 2017.

[39] Mark Steiner. Kripke on logicism, Wittgenstein, and de re beliefs about numbers. In Alan Berger (ed.), Saul Kripke, pages160-176. Cambridge U.P., New York, 2011.

[40] Saharon Shelah. A compactness theorem for singular cardinals, free algebras, Whitehead problem and transversals. Israel J. Math., 21(4):319-349, 1975.

[41] Saharon Shelah. On universal graphs without instances of CH. Annals of Pure and Applied Logic, 26(1):75 - 87, 1984.

[42] Saharon Shelah. Universal graphs without instances of $\mathrm{CH}$ : revisited. Israel Journal of Mathematics, 70(1):69 - 81, 1990.

[43] Saharon Shelah. Cardinal Arithmetic, volume 29 of Oxford Logic Guides. The Clarendon Press Oxford University Press, New York, 1994. Oxford Science Publications.

[44] Jack Silver. On the singular cardinals problem. In Proceedings of the International Congress of Mathematicians (Vancouver, B. C., 1974), Vol. 1, pages 265-268. Canad. Math. Congress, Montreal, Que., 1975. 
[45] Robert M. Solovay. $2^{\aleph_{0}}$ can be anything it ought to be. In J. W. Addison, Leon Henkin, and Alfred Tarski, editors, The theory of models. Proceedings of the 1963 International Symposium at Berkeley, page 435, Amsterdam, 1965. North-Holland Publishing Co.

[46] Robert M. Solovay. Strongly compact cardinals and the GCH. In Leon Henkin, John Addison, William Craig, Dana Scott, and Robert Vaught, editors, Proceedings of the Tarski Symposium, Proceedings of Symposia in Pure Mathematics, Vol. XXV, pages 365-372. Published for the Association for Symbolic Logic by the American Mathematical Society, Providence, R. I., 1974.

[47] S. Walsh. Comparing Peano arithmetic, Basic Law V, and Hume's Principle. Annals of Pure and Applied Logic, 163:1679-1709, 2012.

[48] S Walsh and S Ebels-Duggan. Relative categoricity and abstraction principles. The Review of Symbolic Logic, 8(3):572-606, 2015.

[49] Crispin Wright. Frege's conception of numbers as objects, volume 2 of Scots Philosophical Monograph Series. Aberdeen University Press, Aberdeen, 1983.

[50] Ernst Zermelo. Über Grenzzahlen und Mengenbereiche. Fundamenta Mathematicae, 16:2947, 1930. 